\title{
A RE-LOOK AT MOBILE PHONES USAGE ISSUES
}

\author{
Elisha Mabunda ${ }^{1}$, Edward Chikuni ${ }^{2}$ Rumbidzai Machemedze ${ }^{3}$ \\ ${ }^{1,2,3}$ University of Zimbabwe, Department of Electrical Engineering \\ emabunda50@gmail.com, echikuni@ieee.org,rmachemedze@yahoo.com
}

\begin{abstract}
Mobile phones have become very prominent nowadays and an appreciable world population uses these devices on a daily basis. These mobile phones emit electromagnetic radiation in the radiofrequency range of the electromagnetic spectrum which is around $3 \mathrm{kHz}$ to $300 \mathrm{GHz}$. This type of radiation is called non-ionizing and concerns have been raised in the past concerning its effects on humans. Many researches have been carried out with the aim to determine whether the mobile phones are safe to use or not. This paper reviewed the work of some of these researchers. Focus was put on the methods used by the researchers, sources of their funding, electromagnetic radiation sources used and the number of experiment participants. Results were also compared taking into account the different methodologies used. Much attention was directed to the researches concerning mobile phone radiation on the human head because it is always in close proximity with the phone during phone calls. Quality and accuracy of human head modeling was also reviewed. The human heart, sperm motility and human fetus were also considered. From the results, mobile phone users are recommended to reserve long phone calls for landlines, use hands-free devices e.g. earphones to reduce the proximity between the phone and the head. Switching from GSM to CDMA can also be considered and using a mobile phone only when the network signals are strong is also recommended. The user may also carry the mobile phone somewhere else away from the breast pocket, trouser pocket and pregnant women are encouraged to exercise caution. However, more research needs to be carried out in-order to obtain accurate and clearer results on this issue because past researches produced very inconsistent results.
\end{abstract}

Keywords: Mobile phones, electromagnetic radiation, antennas, Wi-Fi Specific Absorption

\section{Introduction}

A mobile phone also called cellular phone (cell phone) is an electronic device used in telecommunications which does not require wired network but uses wireless network or satellite transmissions. The most basic cell phone provides voice services and short message services (sms) whilst recent models also provide multimedia message services (mms), WI-FI, blue-tooth, internet browsing (mobile data) etc. These are generally referred to as smart phones.

Antennas of mobile phones set up networks with base station antennas for signal transmission to take place. Different mobile phone models use different types of antennas and operate at different frequencies due to the type of information to be transmitted e.g. voice and data. A single mobile phone can also have different antennas each for a specific purpose e.g. primary cellular antenna for transmission and receiving, diversity cellular (receiving only), GPS, WI-FI and near field communication antenna (blue-tooth).

ACRID 2017, June 20-21, Victoria Falls, Zimbabwe

Copyright (C) 2017

DOI 10.4108/eai.20-6-2017.2271017 
Frequencies at which the antenna (basically primary cellular antenna) will work at are determined by the type of network used by the mobile phone which can be global system for mobile communications (GSM), code division multiple access (CDMA), integrated digital enhanced network (iDEN), general packet radio service(GPRS), enhanced data for global evolution (EDGE) etc. Antennas using different networks also may use different frequencies in transmission and receiving but the frequencies might overlap.

Antennas emit electromagnetic radiation during transmission and receiving of signals. Electromagnetic radiation is the transmission of energy in space and it has many applications e.g. x-rays for medical purposes but it can also be harmful to human health hence there is need to investigate the effects of radiation on humans from mobile phones. Given the highly increasing number of mobile phones which is estimated at 5 billion (IARC) [1] in the world, it is a great concern to find out the radiation effects of mobile phones so as to ensure safety of mobile phone

\section{Specific Absorption rate (SAR)}

Specific absorption rate is the rate of deposition of energy per unit mass. It is concerned with the dose of energy absorbed and is measured in Watts $/ \mathrm{kg}$. SAR depends on frequency and it has three regions of exposure which are, sub-resonant range $\left(10^{-2}-10\right)$, whole body resonant range $\left(10-10^{2}\right)$, surface heating range $\left(10^{2}-\right.$ $10^{4}$ ) because the human body responds differently to changing radio frequencies [2]. SAR is the power absorbed by the absorbing mass and it also depends on the type of wave (sinusoidal, triangular, square), and average SAR is the ratio of total power absorbed in the body to the mass in which it is absorbed.

\subsection{SAR calculation}

SAR $=\frac{d}{d t} \cdot \frac{d W}{d m}=\frac{d}{d t} \cdot \frac{d W}{\rho(d V)},[3]$, where dW is incremental energy, dm is incremental mass, $\mathrm{dV}$ is the volume element with a given density $\rho$. Temperature measurements can also be taken disregarding the thermal effects of radiation, conduction and convection.

In terms of temperature increase of absorbing mass, SAR $=\frac{c d T}{D e}[3]$, where dT is temperature rise, $\mathrm{dc}$ is a time interval and $\mathrm{c}$ is a constant.

In terms of the internal electric field in the absorbing mass, SAR $\frac{w \varepsilon}{2 \rho}-E^{2}-\frac{\varepsilon}{2 \rho}-E^{2}$ [3], where $\mathrm{E}$ is the maximum value of internal electric field measured in volt- meters.

\subsection{Mobile phone radiation effects}

Many researches were carried out to determine the effects of SAR on the human body. Various research activities resulted in varying reports some even contradicting depending on who was behind the financing of the research. Some found the evidence of positive effects, some found negative effects and others reported no effects at all. 


\subsection{Positive effects result}

Some of the positive effects of radiofrequency radiation are progress in bone recovery and human sperm movement rise. In the research carried out by Satter in 1999, 19 participants with broken or prolonged recovery of long bones were subjected to low amplitude discrete electromagnetic fields with $0.3 \mathrm{msec} \mathrm{V}$, frequency $80 \mathrm{~Hz}, \mathrm{~B}$ 0.01 to $0.1 \mathrm{~m}$ Tesla. 13 patients finished treatment at 3 and a half months and 11 recovered [4]. Johnson also found the same results in 2001 [5]. In 2006, Iorio did an investigation where human sperms were subjected to extremely-low frequency and extremely-medium $50 \mathrm{~Hz}$ frequency and a square wave of amplitude $5 \mathrm{mT}$ and the effects yielded in the first 3 hours prolonged to 21 hours after exposure. [6]

\subsection{Negative effects result}

The negative effects associated with mobile phone use as found from previous researches include thermal heating, headaches, exhaustion, lightheadedness, sleeping disorders, skin resistance decrease, heart rate inconsistency, blood pressure, weakening of the blood brain barrier, memory loss, lack of attention and Concentration, DNA impairment, chromosome breakdowns, acoustic neuroma, brain cancer etc.A research study carried out by Hirata in 2006 examined the relationship linking the maximum energy deposition per unit mass and maximum temperature rise in tissue blood circulation. 0.13 participants from Sweden and 0.31 participants from Norway were taken and they used mobile phones for work only. The participants felt a heating feeling by the ear to which the phone was held [7].

Another research with participants from Sweden and Norway reported that those who used digital mobile phones had a reduced effect of heating feeling by the ear than their counterparts who used analogue mobile phones. Lena Hillert investigated radiofrequency effects on user -claimed symptoms and field detection after a long time of consistent exposure in 2008 and it was discovered that headaches occurred after exposure [8]. Heinrich did a cross-sectional study involving 3022 children and teenagers randomly chosen from the towns of Germany. The study was computer aided and checking of exposure levels was done by a dosimeter, results indicated that teenagers who used mobile phones suffered irritation and the children suffered headaches, nervousness, light-headedness, fear and sleeping disorders [9].

A study done by Tracy Lightfoot in 2005 showed that memory, mental health and cognitive functions were affected [10]. Ching discovered that skin resistance is reduced by exposure to radiofrequency waves [11]. Nam and fellow researchers carried out a study in 2006.The participants comprised 23 men, 19 women and 21 adolescents who were subjected to $300 \mathrm{~mW}$ radiofrequency waves produced by a CDMA mobile phone for half an hour. The women had no significant reduction in skin resistance but adolescents and men had a reduced skin resistance which was restored in 10 minutes after exposure. Results showed reduced skin resistance because of a rise in sweat production due to stimulus in sensitive nerves [12].

A research carried out by Salford in 1994 confirmed that RF exposure disturbs blood brain barrier functionality [13]. In 2001, Togo examined how heart rate varies during deep sleep using very-low frequency of $(0.003-0.04 \mathrm{~Hz})$ and found very low frequency heart rate variability [14]. Among the negative effects of radio frequency radiation is cancer which is deadly and the seemingly most important to be concerned about. 
Hardell carried out a study on brain cancer risk to rural and urban folk in 2005. Participants were between 20 and 80 years during 1997-2000 in Sweden. It was a case-control study matching sex and age and the results showed a risk in rural folk because digital mobile phones emit more power to cover the distance between base stations than urban folk [15].

The same researcher undertook a case control study using deceased people. 346 people who died of brain tumors were cases and 343 who died of other malignant diseases different from brain cancer as controls. 276 people who also died from diseases other than cancer participated as controls also. The deceased's next of kin were conducted and filled questionnaires about mobile phone use patterns of the deceased for both the participants. The results showed that there is an increased risk of mobile phone use for more than five years [16].

Muscat did an investigation in 2006 and results showed an increased occurrence of acoustic neuroma. It was a hospital-based case control with 90 patients and 86 controls. The users had spent 4.1 years with phone users and controls only 2.2 years [16]. Christensen carried out a research in 2004. The participants were in the range of 20-69 years and had reported cases of glioma and meningioma between 2000 and 2002.Glioma patients were 371 and meningioma patients were 273 , the results showed that a prolonged mobile phone use increased glioma risk [17]. Hung studied the effects of the cell phone during talk mode, silent, and stand-by mode. He found out that mobile phone radiations delay sleep [18].

The Prospective Million Women study carried out investigations on mobile phone users and found out that mobile phone use for more than 5 years causes an increase in the risk of acoustic neuroma. A case control study in Sweden examined two groups under cellphone use, people aged 20-69 and above and those aged below 20 years and the younger group had an increased risk of brain cancer whilst the older group had no link to brain cancer risk and cell phone use.

\subsection{No effects result}

Some of the research studies showed no effects of mobile phone radiation to humans. Myers found no effects of mobile phone radiation in 1990 [19], Zeni examined blood from six voluntary participants in 2008. The blood was exposed to Radiofrequency for 6 minutes and the wash-off period was 2 hours [20]. Silke also carried out a research in 2008 and found no link between radiofrequency waves of frequency 1950 $\mathrm{MHz}$ and energy deposition per unit mass of $2.2 \mathrm{~W} / \mathrm{kg}$. Results did not show any DNA damage, effects to cell movement and micro nucleated cells and human health.329 adults participated and were exposed to RF for 24 hours and no association was found between RF and human health [21]. Cinel examined 496 participants in the doubleblinded research study which considered exhaustion, lightheadedness, warm feelings and skin irritations in 2008 .

The participants were subjected to mobile phone radiofrequency signals for 40 minutes and to sham signals for another 40 minutes.50\% of the group was subjected to mobile phones which use GSM and another $50 \%$ to continuous waves. The results showed no consistency to the exhaustion, lightheadedness, irritation and warmth [22]. Another research was the Interphone study which was carried out by academics from 13 nations. The investigation was on head and neck tumors but there was no clear relationship between cell phone use and the tumors [23]. The 
Cohort study in Denmark obtained information of patients from the country's cancer records office who were mobile phone users and they got 358000 subscribers who had cancer. They carried out an analysis and found no association between cellphone use and malignant ailments [24].

\section{Discussion}

In 1983 mobile phones came into the market without being tested for safety. They were exempted from testing because they were low powered hence it was said that they could not heat living tissue but every device which releases radiation should be tested before getting to the consumer [25].

Researches which produced positive results had small numbers of controls and many studies also were concerned about adults, overlooking children and did not take into account pregnant women, fetus and those who are principally sensitive to electromagnetic fields. Some researches were undertaken on political grounds and some used artificial radiation, some un-modulated sources but mobile phones use pulsed-modulated and amplitude-modulated signals.

Many of the studies were financed by communications corporations so bias is inevitable. 59 studies were selected of which 12 of them were sponsored by the communications industry, 11 were sponsored by charity organizations, 14 received various sponsorships of which communications corporations took part and 22 had no specifications of funding sources [26]

From the report, telecommunications firms had the highest number of funded studies so bias is very possible as their main goal is to remain in business. Odds ratio between charity organizations and telecommunications funded studies was 95\% CI, 0.020.7829]. Cellphone corporations sponsored 7 studies in the US and six of them declared no relationship between cell phone use and cancer.

The INTER-PHONE study (2000) [27] which also found no relationship between human health and mobile phone use was financed by GSM Association and Mobile Manufacturers forum to a greater extent [28]. Both these companies are extensively established in the telecommunications industry and their goal is to grow in business so bias has a very high probability. Universities and hospitals in the United States financed 18 studies and $67 \%$ of them found a relationship between mobile phone use and cancer. This was the main entity to find a relationship between cancer and mobile phone use. This implies that results interpretation from health effects of radiofrequency wave studies should consider the source of funding.

Reports from participants about mobile phone use of a late person from close relative can be wrong especially surveys, even an individual may not recall their phone use patterns accurately. Studies carried out in the $20^{\text {th }}$ century used analogue mobile phones so outcomes can be different now that there are digital mobile phones and advanced technologies in contrast to the early 1990s mobile phone technologies.

\section{Recommendations}

The governments should make sure that mobile phones being sold in their respective countries undergo safety testing. Regulation boards for non-ionizing radiation must be set up or included into those for ionizing radiation especially in Africa because in 
all researches about non-ionizing radiation, Africa seems to be lagging behind. Awareness campaigns through the public, schools, hospitals and workplaces help people to know about the effects which come with mobile phone technologies.

Companies providing mobile phones to the consumers should be monitored by regulation authorities as to whether they stick to safety guidelines in their manufacturing and also to see whether they are testing their devices.

They should also invest in educating the public about all new technologies which come and how to make use of them in safer ways.

All mobile phones should be provided with true SAR values in their manuals to enable the mobile phone users to have full knowledge and minimize their radiation exposure if need be. The public should not be ignorant of awareness and publications so as to stay safe. Technology advancement is a good thing but safety must be the first aspect hence consumers need to be open minded.

\section{Conclusion}

Further research is necessary to get clear consistent results which enable researchers to reach a firm conclusion about the mobile phone radiation effects. Modern methods of measurement, simulation and computation should be used to improve accuracy of results because $20^{\text {th }}$ century researchers carried out experiments using the available methods which are inferior to modern methods so results can come out differently from past research results.

The researches should be carried out by highly qualified and knowledgeable personnel in the subject area and these researchers should not be affiliated to any telecommunications company or government or any entity which might have financial interest in the telecommunications business to ensure integrity of results.

The experiments involved should be carried out using different real mobile phones and human volunteers under electrically tested conditions. The human volunteers should be diverse i.e. albinos, men, women, all world races, children and pregnant women to cater for everyone's safety because people respond differently to the same conditions due to differences in age, skin color, hormones etc.

\section{References}

[1] World Health Organization, International Agency for Research on Cancer, Vol 102,2011

[2] Bohr H, Brunak S, Bohr J, "Molecular wring resonances in chain molecules,"

Bio electromagnetics 18(1997) 187-189

[3] "IEEEC standard for safety level with respect to human exposure to

Radiofrequency field, $3 \mathrm{KHz}-300 \mathrm{KHz}$," Tech. Rep, IEEE c95.1, 1999

[4]Satter Syed S, Islam M S, Rabbani K S, Talukder M s," Pulsed electromagnetic fields for the treatment of bone fractures, "Bangladesh Med Res Counc Bull,25:610,1999 .

[5] Johnson MT, Vanscoy-Covnett A, Vesper DN, Swez JA, Chamberlain JK, Seaward MB, Nindl G, "Electromagnetic fields used clinically to improve bone healing also impact lymphocyte proliferation in vitro," Biomed Scilhstrum,37: 215 $220,2001$.

[6] Iorio R, Soringmaglio R, Gaetano A D, Finetti N, Francavilla F, Santucci R, 
Tettamanti E, Colonna R," A preliminary study of oscillating electromagnetic field effects on human spermatozoon motility," Bio-electromagnetics, June 2006.

[7] Hirata," Peak spatial-average SAR and temperature increase due to antennas attached to human trunk," Journal of the American College of Cardiology, Volume 48, Issue 1, pages 185-186, 2006.

[8] Lena Hillert, Torbjon Akerstedt, "The effects of $884 \mathrm{MHz}$ GSM wireless communication signals on headache and other symptoms: An experimental provocation study, "Bio-electromagnetics, 29:185 - 196, 2008.

[9] Heinrich S, Thomas S, Heumann C, Association between exposure to radiofrequency electromagnetic fields assessed by dosimetry and acute symptoms in children and adolescents: a population based cross sectional study. Environmental Health 25:9(75):1-9, 2001.

[10] Tracy J Lightfoot, Christine F Skibola, "Risk of Non-Hodgkin Lymphoma Associated with Polymorphisms in Foliate-Metabolizing Genes, "Cancer Epidemiology Biomarkers \& Prevention, Vol 14, 2999-3003,2005.

[11] Chang Namki, Sung Woo Kim, "Effects of RF exposure of teenagers and adults by CDMA' cellular phones, Bio-electromagnetics Volume 27 Issue 7, pages 509-514, 2006.

[12] Nam KC, Kim SW, Kim SC, "Effects of RF exposure of teenagers and adults by COMA cellular phones. Bio-electromagnetics 27(7):509-14, 2006.

[13] Salford LG, Brun A, Sturesson K, Eberhard JL, Persson BR," Permeability of the blood- brain barrier induced by $915 \mathrm{MHz}$ electromagnetic radiation, continuous wave and modulated at 8, 16, 50 and $200 \mathrm{MHz}$," Microsc. Res. Tech 15:535-542, 1994.

[14] Togo F, Yamamoto Y," Decreased fractal component of human heart rate variability during non-REM sleep, “Am J Physiol Heart Circ Physiol, 280:H17-H21, Vol 280, Issue 1, H17-H21, 2001.

[15] Hardell L, Carlberg M, Hansson Mild K," Pooled analysis of two case-control studies on the use of cellular and cordless telephones and the risk of benign brain tumors diagnosed during 1997-2003," Int J Oncol, 28:509-518, 2006.

[16] Muscat JE," Handheld Cellular Telephone use and Risk of Brain cancer," JAMA, the journal of American Medical Association 284.23:3001-7, 2006.

[17] Christensen HC, Schuz J, Kosteljanetz M, Cellular telephone use and risk of acoustic neuroma," Am J Epidemiol, 159.277-83, 2004.

[18] Hung CS, Anderson C, Horne JA, McEvoy," Mobile phone 'talk- signal delays EEG -determined sleep onset, "Neuroscience, 421(1):82-86, 2000.

[19] Myers, "ELF exposure has no effects," ACM Transactions on Information Systems, 8(3):289-320, 1990

[20] Zeni O, Schiavoni A, Perrotta A, Fongo D, Deplano M, Scarfi MR," Evaluation of geno-toxic effects in human leukocytes after in vitro exposure to $1950 \mathrm{MHz}$ UMTS radiofrequency field," Bio-electromagnetics 29:177-184,2008.

[21] Silke Thomas, Anja Kuhnlein, Sabine Heinrich, Georg Praml,Dennis Nowak, Rudiger von Kries, Katja Radon, "Personal exposure to mobile phone frequencies and well- being in adults: A cross-sectional study based on dosimetry, "Bioelectromagnetics ,29:463-479, 2008.

[22] Cinel C, Russo R, Boldini A, Exposure to mobile phone electromagnetic fields and subjective symptoms: a double-blind study. Psychosom Med 70(3):345-8, 2008.

[23] Perspect.2011; 119(11): 1534-1538.Institute of Environmental Sciences. 
[24] Friis S, Storm HH. Urban-rural variation in cancer incidence in Denmark 19431987.Eur J Cancer. 1993; 29A (4):538-544. [PubMed].

[25]" Cellphone poisoning of America", page 13, 2008.

[26] Huss A, Egger M, January 2007.Source of funding and results of studies of health effects of mobile phone use: systematic review of experimental studies.

[27] AT\&T, 2010, Verizon (2010). Health and safety information. Retrieved March 17, 2010 from http://www.cancer.org/docroot/AA/99-0.asp.

[28] GSM Association (GSMA). (2010). about us. Retrieved April 12010 from http://www.gsmworld.com/about-us/in 